\title{
Frequency Characteristics of Double-Walled Carbon Nanotube Resonator with Different Length
}

\author{
Jun-Ha LEE ${ }^{1}$, Jeong-Won KANG ${ }^{2}$, Jin-Tae KIM ${ }^{3 *}$ \\ ${ }^{1}$ Department of Computer Engineering, Sangmyung University, Chunan, Chungnam 31066, Korea \\ ${ }^{2}$ Department of Transportation System Engineering, Korea National University of Transportation, Chungjoo, Chungbuk \\ 27469, Korea \\ ${ }^{3}$ Department of Aerospace Software Engineering, Hanseo University, Seosan, Chungnam 31962, Korea \\ cross $^{\text {ref }}$ http://dx.doi.org/10.5755/j01.ms.22.2.12951
}

Received 22 August 2015; accepted 09 December 2015

\begin{abstract}
In this paper, we have conducted classical molecular dynamics simulations for DWCNTs of various wall lengths to investigate their use as ultrahigh frequency nano-mechanical resonators. We sought to determine the variations in the frequency of these resonators according to changes in the DWCNT wall lengths. For a double-walled carbon nanotube resonator with a shorter inner nanotube, the shorter inner nanotube can be considered to be a flexible core, and thus, the length influences the fundamental frequency. In this paper, we analyze the variation in frequency of ultra-high frequency nano-mechnical resonators constructed from DWCNTs with different wall lengths.

Keywords: molecular dynamic, DWCNT, nanotube resonator, ultra-high frequency, different wall.
\end{abstract}

\section{INTRODUCTION}

Many studies have been conducted on CNTs since the discovery of the carbon nanotube (CNT) in 1991. Recently, the mechanical movement of single-walled or multi-walled CNTs has been actively investigated because CNTs can be applied as a basic material to develop nanosized devices, nano-electronic circuits, and nano-composite materials [1].

CNTs have excellent mechanical, electrical, thermal, and chemical characteristics, and as a result, it is expected for CNTs are be used as next-generation materials in a number of industries, including IT, alternative energy, and composite materials.

Molecular dynamics simulations are extensively used these days because it is very difficult to conduct actual experiments at the nano-scale [2]. Therefore, computer simulations are drawing attention as next-generation experimental platforms. Mechanical resonators play a key role [3] because if the size of the resonator is reduced, the frequency of resonant increases and less energy needs to be consumed [4]. For sensors, high resonance frequencies are synonymous with a high sensitivity [5], and in wireless communication, high-frequency resonators are applicable in high-frequency filters, oscillators, and mixers (among others).

New studies have been conducted in these areas as high-frequency nano-electromechanical systems are further developed, and resonators and oscillators that use CNTs have been recently investigated $[6,7]$. The vibrations of CNTs and the amplitude of the thermal vibrations of a CNT with a tip hanging from the wall are currently being studied to predict the Young's modulus of the CNT [8].

CNTs possess ideal characteristics, such as a high elastic modulus and thermal conductivity, and these ideal

\footnotetext{
* Corresponding author. Tel.: +82-41-660-1364; fax: +82-41-660-1119. E-mail address: jtkim@hanseo.ac.kr (J.T. Kim)
}

characteristics along with their nano-meter size and perfect atomic structure, make CNTs a promising material to build components for high-frequency oscillators targeting nanoelectromechanical systems for signal processing applications.

Double-walled carbon nanotube (DWCNT) resonators have different mechanical structures than SWCNT resonators due to the interlayer interactions that are characterized by the van der Waals $(v d W)$ potential for carbon. Jiang et al. [2] used classical MD simulations to study the energy dissipation of cantilevered singe-walled CNT oscillators. A DWCNT can be fabricated with a short outer wall by thinning and opening the DWCNT through oxidation with carbon dioxide [3] and by burning the outer wall using an electric current. A DWCNT with a short inner wall can be fabricated through the nano peapod-toDWCNT transition [9].

A short inner CNT functions for the DWCNT resonators, and their basic frequency is affected by their length, so this paper proposes a new method to analyze the frequency of a nano-sized resonator using DWCNTs. The proposed method for frequency analysis is expected to be widely applicable for implementation with various frequency devices that control the length of inner or outer CNTs. Furthermore, the proposed method analyzes the ultrahigh frequency characteristics of the nano-sized resonators by applying atomic unit modeling to DWCNTs with different lengths. The results are then analyzed to apply this method for devices of various frequencies. The results of the analysis indicate that the DWCNT resonators with various wall lengths can be used to develop design guidelines to produce very fine synchronizers [10-13].

\section{FREQUENCY CHARACTERISTICS}

Jiang et al. carried out simulations of classical molecular dynamics (MD) to investigate the energy consumption from the oscillations of CNTs with a hanging 
tip [7, 14]. Furthermore, studies have reported results on the vibration characteristics of DWCNT resonators using the continuum model method $[15,16]$. However, these studies used DWCNTs that have the same lengths of the inner and the outer CNTs, and in fact, resonators based on the CNTs can use DWCNTs with different CNT lengths $[17,18]$. However, no studies have yet been conducted for DWCNTs with different lengths of inner and outer CNTs [19-21].

In this paper, we performed the MD simulations aimed at different ends hanging DWCNT resonator has the length (cantilever mode). Accurate experiments on the inter-wall distance of DWCNTs have indicated that this value can change from $3.0 \AA$ to $5.4 \AA$ [22]. However, the CNT distance that offers a high level of stability is mainly $3.5 \AA$ [23]. This study uses 5.0 and 14.0 DWCNTs to analyze the properties according to the different lengths with a CNT space of $3.4 \AA$. This CNT space was selected because it is the best size to produce stable DWCNTs, and as a result, new information has been discovered regarding the basic frequency according to differences in the CNT length [24-27].

The MD simulations were conducted for two cases. In the first case, the inner length (5.0) CNT was fixed at $5 \mathrm{~nm}$ (LI), and the outer length (14.0) CNT changed. In the second case, the outer (5.0) CNT was fixed at $5 \mathrm{~nm}$ while the inner (14.0) length changed from 0 to $10 \mathrm{~nm}$.

It is important to use an appropriate atomic interaction model equation for molecular dynamics simulations. The atomic combination between carbon atoms in a singlewalled carbon nanotube forms a covalent bond that can generally be calculated using the Tersoff-Brenner potential, and so the atomic interaction model equation is the most important consideration for the model in the molecular dynamics simulation [28].

The remote carbon interaction between the CNT walls is the van der Waals interaction, which can be modeled using the Lennard-Jones $12-6$ potential. The TersoffBrenner potential was used because the carbon atom maintains a covalent bond in the CNT [29], and furthermore, the Lennard-Jones $12-6$ (LJ12-6) potential was used for the remote reaction of the carbon atoms while the data derived by Ulbricht was used for the parameters [30].

\section{RESULTS OF ANALYSIS}

Fig. 1 and Fig. 2 show the resonant frequencies as a function of $L_{5}$ and $L_{10}$, and the contour of the resonant frequencies for $L_{5}$ and $L_{10}$, respectively. As shown in Fig. 1 and Fig. 2, a peak in the resonant frequencies occurs for about $1 \mathrm{~nm}$ of DWCNT. The resonant frequencies are thus further reduced depending on the increase in length of $L_{5}$ and $L_{10}$. The resonant frequency curve of the ridge thus produces a peak at $L_{10}=7 \mathrm{~nm}$.

The resonance frequencies are almost constant when $L_{5} \leq L_{10}$, whereas decreasing resonance frequencies follow the frequency decrease trend of SWCNTs with increasing $L_{5}$ when $L_{5}>L_{10}$. These results show that when the inner wall is entirely encapsulated inside the outer wall, the vibration of the DWCNT is greatly influenced by the vibration of the outer wall. However, we note that the encapsulated inner wall is slightly influenced by the vibration of the corresponding DWCNT with the vdW interaction.

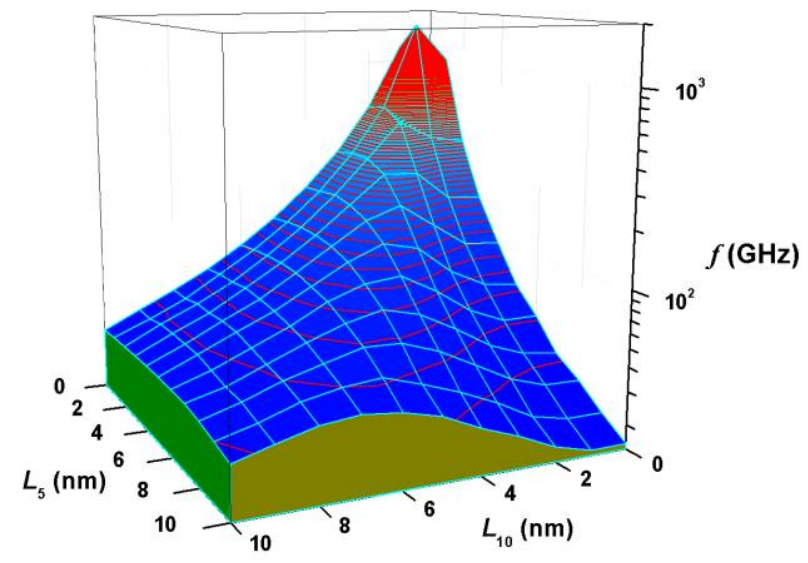

Fig. 1. Resonant frequencies as functions of $L_{5}$ and $L_{10}$

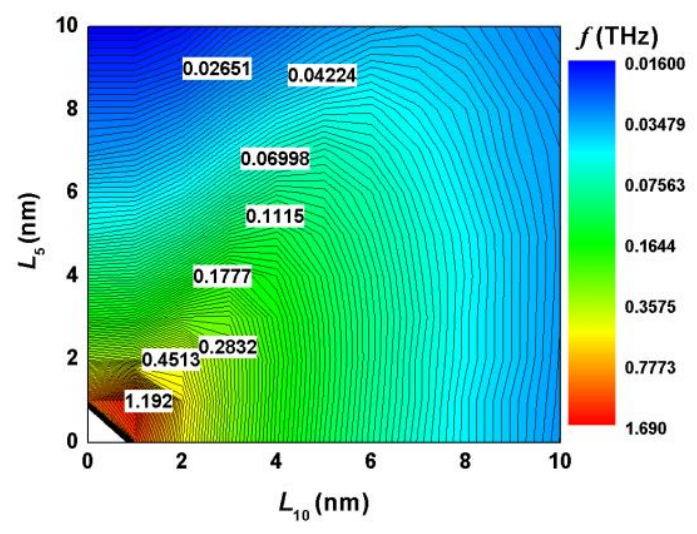

Fig. 2. Contour of the resonant frequencies of $L_{5}$ and $L_{10}$

\section{DISCUSSION}

Fig. 3 shows the displacement of the tip according to the MD time when $L_{I}=5 \mathrm{~nm}$ and $L_{O}$ change from 0 to $10 \mathrm{~nm}$. Fig. 4 shows the displacement of the tip according to the MD time when $L_{O}=5 \mathrm{~nm}$ and $L_{I}$ changes.

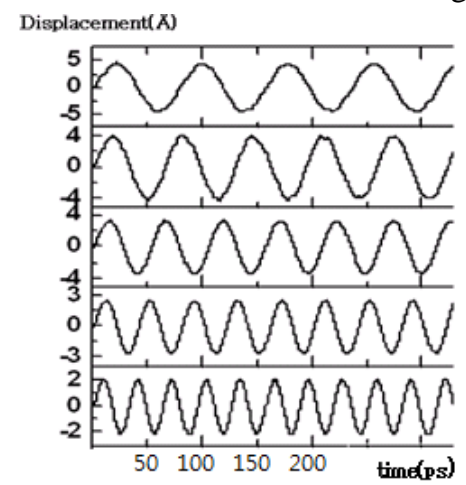

Fig. 3. Displacement MD time when $L_{O}$ changes from 0 to $10 \mathrm{~nm}$

The frequency change due to the differences in the CNT lengths corresponding to Fig. 5 and Fig. 6. In order to induce a basic frequency according to change in the length, a frequency spectrum was obtained from data in Fig. 5 and Fig. 6 using the Fast Fourier Transform (FFT) as in Fig. 5 and Fig. 6. As is shown in Fig. 5, when $L_{I}=5 \mathrm{~nm}$, the basic frequency was observed to decrease as $L_{O}$ exceeded $4 \mathrm{~nm}$. However, as in Fig. 6, the basic frequency remained 
constant according to the increase in $L_{I}$ up to $L_{I}=4 \mathrm{~nm}$ when $L_{O}=5 \mathrm{~nm}$ and decreased when $L_{I}$ was longer than $5 \mathrm{~nm}$.

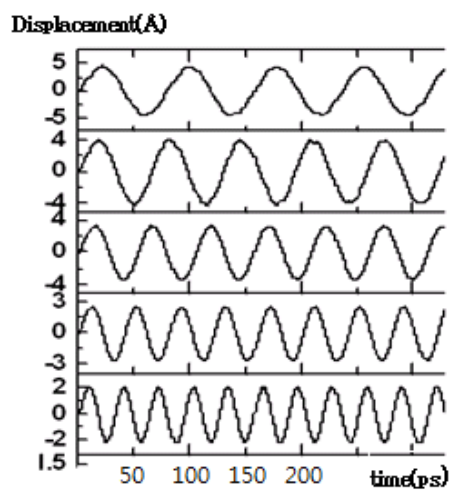

Fig. 4. Displacement MD time when $L_{I}$ changes from 0 to $10 \mathrm{~nm}$

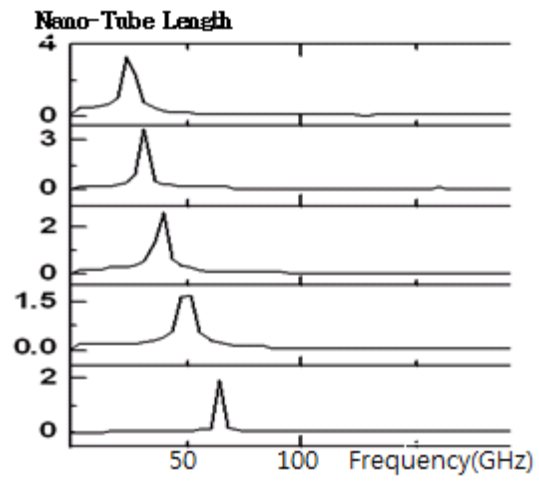

Fig. 5. Spectrum according to the increase in $L O$

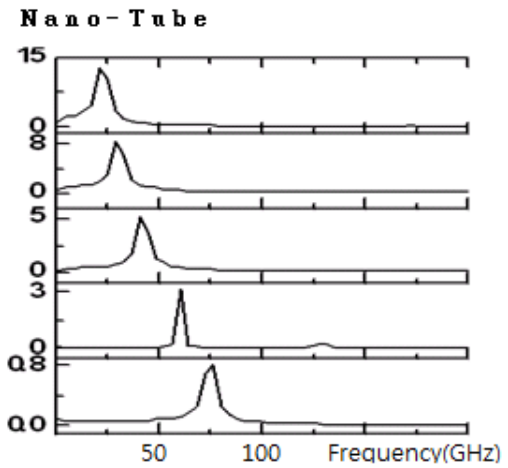

Fig. 6. Spectrum according to the increase in $L_{I}$

Fig. 7 shows the fundamental frequency according to the ratio of $L_{I}$ or $L_{O}$. The fundamental frequency of the (14.0) CNT with an end of $5 \mathrm{~nm}$ length was almost twice that of the (5.0) CNT of the same length. In the case of the external (14.0) CNT with a length of $5 \mathrm{~nm}$, the fundamental frequency increased moderately following the increase in the length of the interior (5.0) CNT to up to $L_{I}=4 \mathrm{~nm}$, but it decreased afterward. When $L_{I}=5 \mathrm{~nm}$, the trend in the frequency change conformed well to the Gauss distribution when the length of $L_{O}$ was between $2-7 \mathrm{~nm}$. In the case of the interior (5.0) CNT, the fundamental frequency increased moderately following the increase in the length of the external (14.0) CNT to up to $L_{O}=4 \mathrm{~nm}$ but decreased afterward. According to the results the regression analysis of the MD simulation using the Gauss distribution, the maximum frequency of the (5.0)(14.0) CNT resonator with a length exceeding $5 \mathrm{~nm}$ was obtained when $L_{O}=3.8 \mathrm{~nm}$ and $L_{I}=5 \mathrm{~nm}$. When $L_{I}$ or $L_{O}$ become longer than $5 \mathrm{~nm}$, all of the frequencies showed a similarity despite their structural differences.

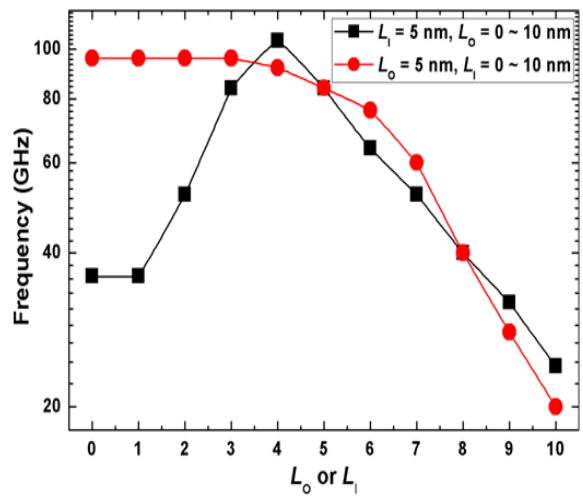

Fig. 7. Fundamental frequency analysis of the Nanotube resonator

For the structure used in the simulations of this paper, one of the corners of the interior and exterior walls is fixed and the other side moves freely. Hence, when the length of the exterior wall exceeds that of the interior wall, the shorter interior wall affects the vibration of the exterior wall. As a result, the fundamental frequency of the short interior wall increased slightly.

\section{CONCLUSIONS}

We conducted classical molecular dynamics simulations to investigate the behavior of ultrahigh frequency nano-mechanical resonators made of DWCNTs with various wall lengths, and we intended to determine the variations in the frequency of these resonators according to the DWCNT wall lengths. In this study, the changes in the basic frequency were analyzed for the DWCNT resonators with tips hanging from the wall and with different CNT lengths. MD simulations were conducted for 5.0 and 14.0 DWCNTs with a $10 \mathrm{~nm}$ or smaller length in order to analyze the basic frequency according to the changes in the DWCNT resonator. The results of the analysis were interpreted such that the DWCNT resonators can show various changes in their performance according to differences in chiral indices. The MD simulations indicate that devices with various frequencies can be implemented when the inner or outer CNT lengths change. Regarding a DWCNT resonator with a CNT that has a short outer part, the free tip plays a key role in the vibration of the long inner CNT. Meanwhile the short inner CNT acted as a soft core in all three boundary conditions, confirming that the basic frequency is affected by the length.

\section{REFERENCES}

1. Li, C., Chou, T.W. Single-Walled Carbon Nanotubes as Ultrahigh Frequency Nanomechanical Resonators Physical Review B 68 2003: pp. 073405-1-073405-1.

2. Jiang, H., Yu, M.F., Liou, B., Huang, Y. Intrinsic Energy Loss Mechanisms in a Cantilevered Carbon Nanotube Beam Oscillator Physical Review Letters 93 2004: pp. 185501-1 - 185501-4.

3. Tsang, S.C., Harris, P.J.F., Green, M.L.H. Thinning and Opening of Carbon Nanotubes by Oxidation Using Carbon Dioxide Nature 362 1993: pp. 520-522. 
4. Collins, P.G., Hersam, M., Arnold, M., Martel, R., Avouris, P. Current Saturation and Electrical Breakdown in Multiwalled Carbon Nanotubes Physical Review Letters 86 2001: pp. 3128-3131

5. Tersoff, J. Modeling Solid-State Chemistry: Interatomic Potentials for Multicomponent Systems Physical Review B 39 1989: pp. 5566-5568.

6. Li, C., Thostenson, E.T., Chou, T.W. Sensors and Actuators Based on Carbon Nanotubes and Their Composites: A Review Composites Science Technology 68 2008: pp. $1227-1249$.

http://dx.doi.org/10.1016/j.compscitech.2008.01.006

7. Ulbricht, $\mathbf{H}$., Moos, G., Hertel, T. Interaction of $\mathrm{C}_{60}$ with Carbon Nanotubes and Graphite Physical Review Letters 90 2003: pp. 095501-1-095501-4.

8. Qian, D., Wagner, G. J., Liu, W.K., Yu, M.F., Ruoff, R.S. Mechanics of Carbon Nanotubes Applied Mechanics Reviews 55 2002: pp. 495-533.

http://dx.doi.org/10.1115/1.1490129

9. Guisca, C.E., Tison, Y., Stolojan, V., Borowiak-Palen, E., Silva, S.R.P. Inner-Tube Chirality Determination for Double-Walled Carbon Nanotubes by Scanning Tunneling Microscopy Nano Letters 7 2007: pp. 1232-1239.

10. Kwon, O.K., Lee, J.H., Park, J.C., Kim, K.S., Kang, J.W. Molecular Dynamics Simulation Study on GrapheneNanoribbon-Resonators Tuned by Adjusting Axial Strain Corrent Applied Physics 13(2) 2013: pp. 360-365.

11. Kim, J. T., Lee, J. H. Frequency Vibrational Behavior Analysis of Double-Wall Carbon Nanotube Resonator Journal of the Korea Institute of Information and Communication Engineering 15 2011: pp. 169-174.

12. Kim, J.S., Kim, G.H., Kim, C.I. Synthesis and Microstructure of Single-Walled Carbon Nonotubes By Catalytic Chemical Vapor Deposition Method The Transactions of The Korean Institute of Electrical Engineers 55C 2006: pp. 359-363.

13. Jung, S.I., Lee, S.B. A Reliable Field Emission Performance of Double-Walled Carbon Nanotube Field Emitters Journal of the Korean Vacuum Society 17 2008: pp. 566-575.

14. Zhao, X., Cummings, P. T. Molecular Dynamics Study of Carbon Nanotube Oscillators Revisited The Journal of Chemical Physics 124 2006: p. 134705-1-134705-8.

15. Yakobson, B.I., Brabec, C.J., Bernholc, J. Nanomechanics of Carbon Tubes: Instabilities Beyond Linear Response Physical Review Letters 76 1996: pp. 2511-2514. http://dx.doi.org/10.1103/PhysRevLett.76.2511

16. De Los Santos, H.J. Introduction to Microelectromechanical Microwave Systems, Artech House Publishers, London, 1999.

17. Kang, J.W., Choi, Y.G., Kim, Y.H., Jiang, Q., Kwon, O.K., Hwang, H.J. The Frequency of Cantilevered DoubleWall Carbon Nanotube Resonators as a Function of Outer Wall Length Journal of Physics Condensed Matter 21 2009: p. $385301-385301$.
18. Kang, J.W., Kwon, O.K. A Molecular Dynamics Simulation Study on Resonance Frequencies Comparison of Tunable Carbon-Nanotube Resonators Applied Surface Science 258 (6) 2012: pp. 2014-2016. http://dx.doi.org/10.1016/j.apsusc.2011.05.026

19. Lair, S.L., Herndon, W.C., Murr, L.E. Stability Comparison of Simulated Double-Walled Carbon Nanotube Structures Carbon 46 2008: pp. 2083-2095.

20. Tersoff, J. New Empirical Model for the Structural Properties of Silicon Physical Review Letters 56 1986: pp. 632-635.

21. Brenner, D.W. Empirical Potential for Hydrocarbons for Use In Simulating The Chemical Vapor Deposition of Diamond Films Physical Review B 42 1990: pp. $9458-9471$.

22. Jensen, K., Kim, K., Zettl, A. An Atomic-Resolution Nanomechanical Mass Sensor Nature Nanotechnology 3 2008: pp. $533-537$. http://dx.doi.org/10.1038/nnano.2008.200

23. Sazonova, V., Yaish, Y., Ustunel, H., Roundy, D., Arias, T.A., McEuen, P.L. A Tunable Carbon Nanotube Electromechanical Oscillator Nature 431 2004: pp. 284-287.

24. Song, Y.J., Lee, J.H., Kim, J.T., Choi, J.H. A Study of Ultra-High Frequency Characteristics of Twin-Wall Carbon Nanotube Resonator Proceedings of International Conference on Electronics, Information and Communications 2010: pp. 417-418.

25. Kang, J.W., Kwon, O.K., Hwang, H.J., Jiang, Q. Resonance Frequency Distribution of Cantilevered (5.5)(10.10) Double-Walled Carbon Nanotube With Different Lengths Molecular Simulation 37 (1) 2011: pp. $18-22$.

26. Lee, J.H., Kim, K.S., Kang, J.W. Developing A NanotubeBased Electromechanical-Device for Measuring Angular Velocity Computational Materials Science 48 2010: pp. 837-841.

27. Kang, J.W., Kwon, O.K., Lee, J.H., Choi, Y.G., Hwang, H.J. Frequency Change by Inter-Walled Length Difference of Double-Wall Carbon Resonator Solid State Communications 149 2009: pp. 1574-1577. http://dx.doi.org/10.1016/j.ssc.2009.05.041

28. Zheng, Q., Jiang, Q. Multiwalled Carbon Nanotubes as Gigahertz Oscillators Physical Review Letters 88 2002: pp. 045503-1-045503-3.

29. Treacy, M.M.J., $\quad$ Ebbesen, T.W., Gibson, T.M. Exceptionally High Young's Modulus Observed for Individual Carbon Nanotubes Nature 381 1996: pp. 678-680.

30. Xu, K.Y., Guo, X.N., Ru, C.Q. Vibration of A DoubleWalled Carbon Nanotube Aroused by Nonlinear Intertube Van Der Waals Forces Journal of Applied Physics 99 2006: pp. 064303-1-064303-7. 